\title{
Modelling of Charging Demand for Electric Vehicle based on Person-trip Survey Data
}

\author{
T. Kato ${ }^{1}$, T. Matsuki ${ }^{2}$, M. Imanaka ${ }^{1}$, M. Kurimoto ${ }^{1}$ and S. Sugimoto ${ }^{1}$ \\ ${ }^{1}$ Institute of Materials and Systems for Sustainability \\ ${ }^{2}$ Department of Electrical Engineering, School of Engineering \\ Nagoya University \\ Furo-cho, Chikusa-ku, 464-8603 Nagoya (Japan) \\ Phone number: +81 52789 5373, e-mail: tkato@imass.nagoya-u.ac.jp
}

\begin{abstract}
In electricity distribution networks, voltage might be dropped significantly by simultaneous charging of high penetration Electric Vehicle (EV). The impact of EV on voltage drop can be different among areas depending on various factors such as original electricity demand profile without EV, EV penetration level and usage pattern, etc. For the statistical assessment of impact of high penetration EV in various distribution networks, this study proposes a model to estimate time-series data of electricity demand including EV charging. The proposed model is based on Grid Square Statistics Data on geographical distribution of population, employee, etc. and Person-trip Survey Data. As an example using the proposed model, this study compares three EV charging strategies in suburban area of Nagoya, Japan. As a result, if the EV charging starts simultaneously at 23:00 at which the night-time discount electricity rate is applied, the night-time electricity demand can be much larger than the day-time peak demand without EV. The increase can be mitigated by autonomous charging control according to the time and SOC of EV returning home in most areas. Finally, by using the calculated electricity demand profile, this study assesses the impact of EV charging on voltage profile. The result shows that the impact of EV charging on distribution network voltage can be avoided by applying the proposed autonomous charging control scheme.
\end{abstract}

\section{Key words}

Electric vehicle, electricity distribution network, electricity demand, statistical data, person-trip survey data

\section{Introduction}

In a future electricity distribution network with high penetration of Electric Vehicle (EV), the significant voltage drop can occur when the high penetration EV is charged simultaneously after returning home in the evening. There are various studies regarding the impact of EV penetration on voltage control in a distribution network and proposals of voltage control to avoid voltage drop. In some studies, EV is controlled to support voltage control[1]-[5]. The impact of EV on the voltage drop and the contribution of newly proposed control schemes would be different among areas depending on various factors such as original electricity demand profile without EV, EV penetration level and usage pattern, etc. Therefore, the modelling of electricity demand profile including EV charging contributes to investigate a suitable voltage control scheme in future electricity distribution networks.

For this purpose, this study develops a model to estimate time-series data of electricity demand including EV charging in an arbitrary distribution network based on Grid Square Statistics Data on geographical distribution of population, employee, etc. and Person-trip Survey Data. First, the calculation procedures of the proposed model is explained. Then, as an example of application of proposed model, the electricity demand profiles including EV charging in three strategies are compared. Finally, the impact assessment of EV charging on voltage profile is demonstrated.

\section{Modelling of Electricity Demand in Each 500 m Grid Square [6]}

Electricity demand estimation model used in this study is the modification to the model developed in our previous work[6]. The proposed model estimates hourly value of the electricity demand for a year in an arbitrary distribution network. The electricity demand at middle and low voltage mainly in residential and commercial sectors is estimated, while the electricity demand receiving at special high voltage mainly in industrial sector is out of concern. Because the model is based on $500 \mathrm{~m}$ grid square statistics, the spatial resolution of demand is $500 \mathrm{~m}$.

The electricity demand in mesh-code $m D^{m}(t)$ is calculated by using following equation.

$$
D^{m}(t)=\sum_{j=1}^{8} \sum_{k=1}^{4}\left\{\frac{d_{j}^{k}(t) \times F_{j}^{m} \times S_{j}^{k}}{\eta_{j}^{k}}\right\}+D_{I}^{m}(t)+D_{S}^{m}(t)
$$


where $j$ : consumer type (detached house, apartment house, single-room house, office, OA-office, store, hotel, hospital, restaurant, school). $k$ : energy usage purpose (power, space-heating, space-cooling, hot-water), $\boldsymbol{d}_{j}^{\boldsymbol{k}}(\boldsymbol{t})$ : unit energy load per floor space, $\boldsymbol{F}_{\boldsymbol{j}}^{\boldsymbol{m}}$ : total floor space in mesh-code $m, \boldsymbol{S}_{\boldsymbol{j}}^{\boldsymbol{k}}$ : sheare of electricity, $\boldsymbol{\eta}_{\boldsymbol{j}}^{\boldsymbol{k}}$ : efficiency of electricity appliance. $\quad \boldsymbol{D}_{I}^{\boldsymbol{m}}(\boldsymbol{t})$ : industrial electricity demand, $\boldsymbol{D}_{S}^{m}(\boldsymbol{t})$ : electricity demand with special contract.

The total floor space of detached house, apartment house and single-room house is calculated based on the number of households categorized into 6 types according to total floor space. The total floor space of commercial building is calculated based on the number of employee and typical floor space per employee. $\boldsymbol{D}_{I}^{m}(\boldsymbol{t})$ and $\boldsymbol{D}_{S}^{m}(\boldsymbol{t})$ is simply calculated by distributing the total demand in utility service area in the past record of actual sales to each grid square in consideration of the land-use statistics and spatial distribution of primary, respectively. The time profile of these data is calculated based on the simply assumed time of use profile.

\section{Aggregated Electricity Demand Profile in Distribution Network [7]}

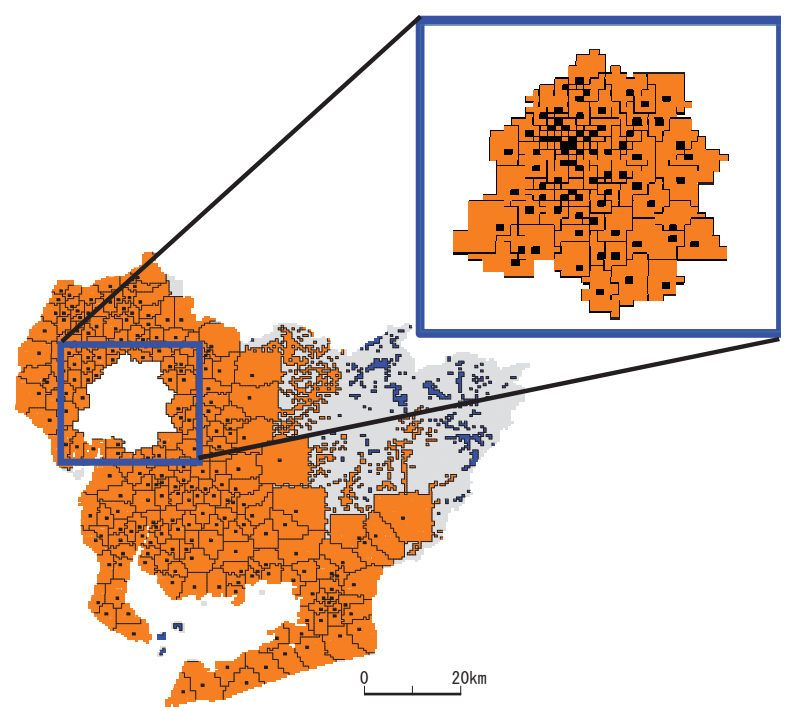

Fig. 1 Estimated territory of distribution networks in Aichi Prefecture, Japan
The location of substaion is determined based on the satellite image, etc., so that the model can be developed based on the publically available data set only. The electricity supply territory of each substation is determined by adding the adjacent $500 \mathrm{~m}$ grid square of substation location one by one in the acceending order of the predetrmined number of substiaon. When the number of grid square added to the territory reaches to the predetermined maximum number (440 squares), or all the grid squares surrounding the territory is already included in other neighboring territories, the adding process is finished. In a rural area, far located grid square from the substaion is added in the additional pcocedure. The details of process can be found in our preceeding study[7].

Figure 1 shows the estimated territory of 298 distribution networks in Aichi Prefecture, Japan. As discussed in [7], the estimated annual maximum electricity demand in each territory varies around $20-40 \mathrm{MW}$, which corresponds to the typical size of distribution substation in Japan. Therefore, the proposed method based on the publically available data set seems to work well to determine the territory of distribution network.

The electricity demand in each distribution network is calculated by summing up the estimated electricity demand in $500 \mathrm{~m}$ grid square included in each distribution network. In order to evaluate the performance of proposed model, the electricity demand in three distribution network territories is compared with the observed electricity demand for typical area i.e. residential area, residences and factories complex area, and rural area in Aichi Prefecture, Japan. Figure 2 shows examples of estimated electricity demand in August in three distribution networks. The black line is the monthly average value of observed electricity demand in weekdays in each distribution network, which was provided by the local electric power company in this area. The observed electricity demand includes the electricity supply of high penetration photovoltaic power generation (PV) as a negative electricity demand. Therefore, the original electricity demand is calculated by estimating the PV power output based on the estimated PV capacity in the record of Feed-in-Tariff scheme and the time series data of ground observation of irradiance at the nearest meteorological observation point of Japan Meteorological

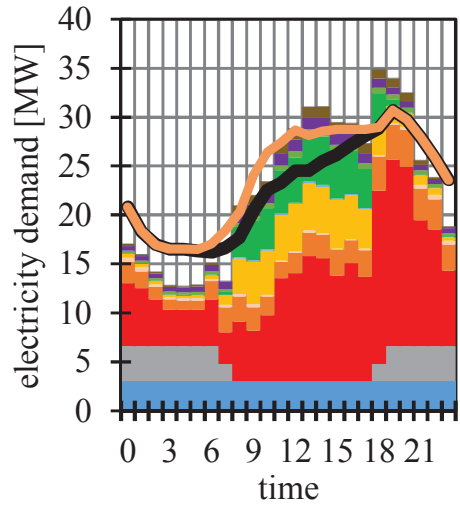

(a) residential area

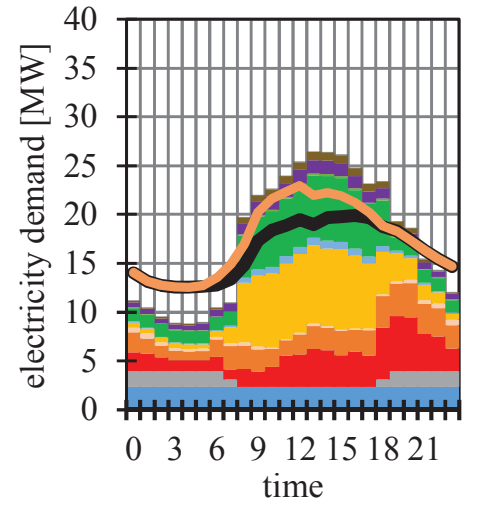

(b) residence and factory area

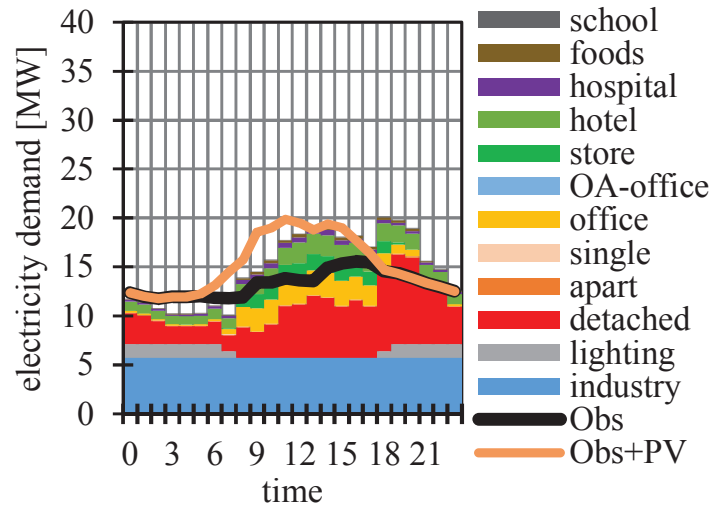

(c) rural area

Fig. 2 Monthly average of estimated electricity demand on weekday in August 
Agency (JMA).

As shown in Figure 2, the proposed method seems well working to estimate the time-series of electricity demand excepting midnight. The monthly mean absolute error (MAE) relative to the total electricity demand varies in $6 \%-15 \%$ depending on month in every area. Although the midnight electricity consumption should be increased by considering low electricity rate in midnight, the estimation accuracy seems acceptable level for the first order analysis of future electricity demand by the high penetration of EV.

\section{Analysis of Person-trip Data}

The proposed model estimates vehicle utilization patterns based on "Person-trip survey data", which is provided every five years for some metropolitan areas in Japan. A person-trip is a trip from one address to another by one person using any mode of transportation, for various purposes of trip. In order to categorize the destination in a simple way, the data is summarized in twenty large zones. Each large zone consists of several numbers of middle zones, and each middle zone consists of several numbers of small zones. Table 1 shows five categories of destination zone used in this study.

Focusing on the commuting purpose, this study calculated the ratio of destination zones in five categories on average of small zones in thirteen large zones among twenty large zones in the person trip data. Figure 3 shows the result. Large zones \#1 - \#5 are located in Nagoya City with more than more than 2.5 million population. Because the industrial area locates mainly around Nagoya City, therefore, the ratio of destination category $\mathrm{E}$ tends to be larger in these large zones than other large zones.

Figure 4 shows the cumulative ratio of returning home from commuting along with the time in a day on average in small zones for thirteen large zones. The returning ratio increases at 16:30 - 17:00 depending on the location of large zone. More than $60 \%$ returns from the commuting between 17:00 and 21:00. Because the residential electricity demand in such a time zone is the largest in a day, if the EV charging starts just after the returning home, the electricity demand including EV charging can be significantly large.

Figure 5 shows the cumulative ratio of leaving home for commuting along with the time in a day on average in small zones for thirteen large zones. The leaving home ratio increases around 7:00 in many large zones. This means that the EV charging should be finished before 7:00 if $\mathrm{EV}$ is used for the commuting.

\section{Modelling of EV Charging}

Figure 6 shows the relation between the number of household and the number of vehicles in 70 municipalities in Aichi Prefecture. Based on this relation and the assumption on future EV penetration shown in some governmental reports in Japan, this study assumes that the
Table 1 Destination zone in 5 categories

\begin{tabular}{|c|l|}
\hline category & \multicolumn{1}{|c|}{ Destination } \\
\hline A & within same small zone \\
\hline B & other small zone in same middle zone \\
\hline C & neighboring middle zone in same large zone \\
\hline D & non-neighboring middle zone in same large zone \\
\hline E & other large zone \\
\hline
\end{tabular}

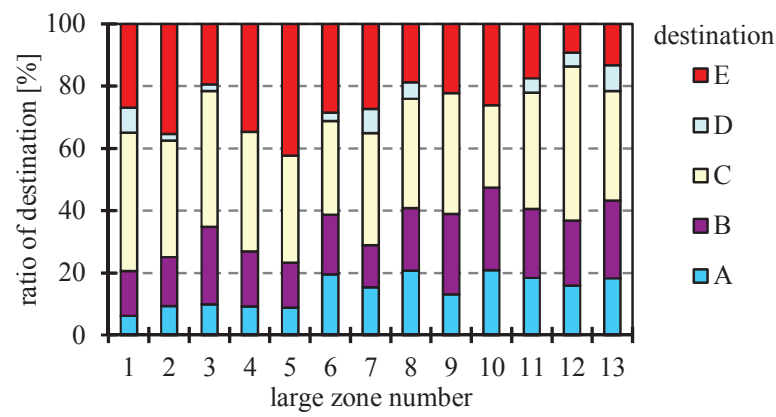

Fig. 3 Ratio of destination zones in five categories on average of small zones in thirteen large zones
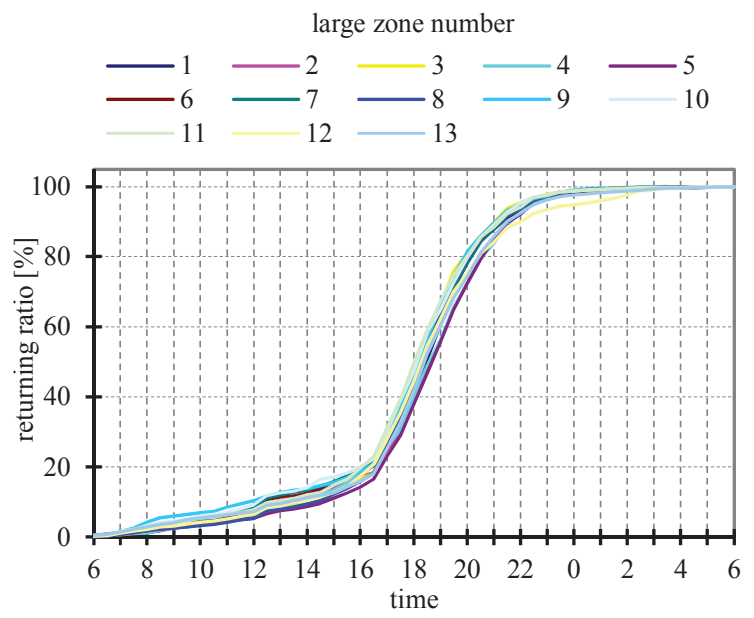

Fig. 4 Cumulative ratio of returning home from commuting along with the time in a day on average in small zones for thirteen large zones
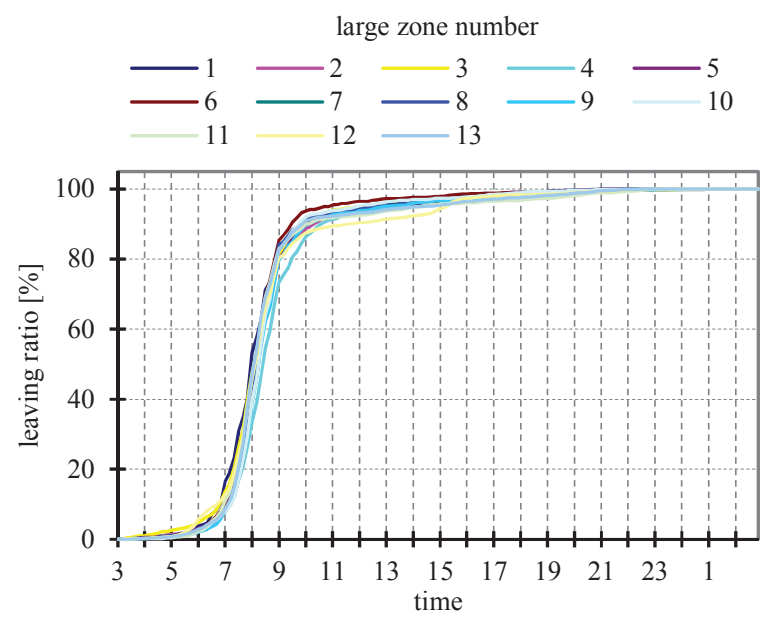

Fig. 5 Cmumulative ratio of leaving home for commuting along with the time in a day on average in small zones for thirteen large zones

number of $\mathrm{EV}$ is $16 \%$ of households in each distribution network. 


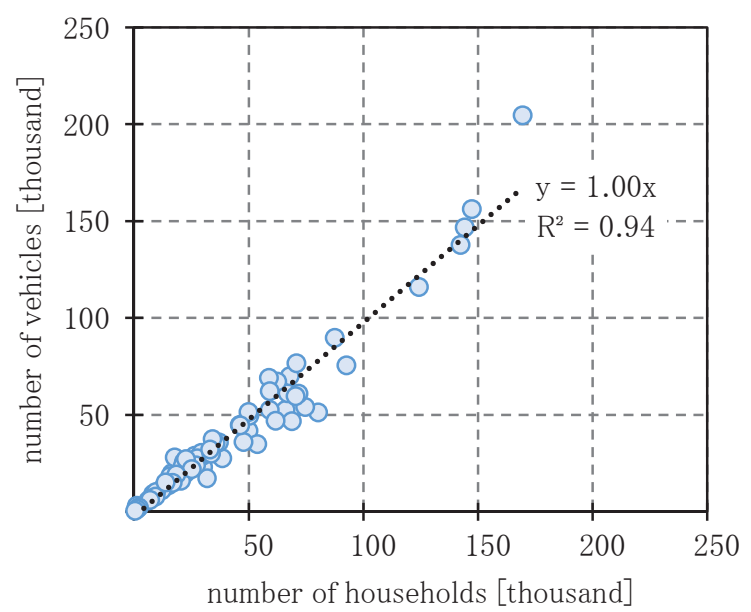

Fig. 6 Relation between number of households and vehicles in each municiparity

Table 2 Assumption on round-trip distance for commuting by vehicle depending on destination zone for commuting

\begin{tabular}{|l|r|r|r|r|r|}
\hline \multirow{2}{*}{$\begin{array}{l}\text { large zone } \\
\text { number }\end{array}$} & \multicolumn{6}{|c|}{ destination } \\
\cline { 2 - 6 } & \multicolumn{1}{|c|}{$\mathrm{A}$} & \multicolumn{1}{|l|}{ B } & \multicolumn{1}{l|}{ C } & \multicolumn{1}{l|}{ D } & \multicolumn{1}{l|}{ E } \\
\hline 1 & 1.5 & 3.9 & 8.7 & 6.6 & 10.5 \\
\hline 2 & 1.7 & 4.8 & 9.9 & 6.3 & 18.4 \\
\hline 3 & 2.0 & 5.7 & 12.1 & 8.9 & 16.9 \\
\hline 4 & 2.4 & 6.0 & 14.3 & 6.4 & 17.5 \\
\hline 5 & 2.4 & 6.2 & 12.2 & 6.7 & 25.7 \\
\hline 6 & 4.0 & 9.0 & 17.5 & 11.8 & 26.6 \\
\hline 7 & 3.4 & 6.4 & 13.5 & 13.3 & 27.7 \\
\hline 8 & 3.8 & 7.7 & 16.3 & 13.1 & 27.9 \\
\hline 9 & 3.5 & 7.8 & 15.5 & 8.4 & 23.4 \\
\hline 10 & 5.0 & 8.8 & 18.2 & 10.0 & 23.0 \\
\hline 11 & 4.5 & 7.7 & 14.4 & 17.2 & 22.8 \\
\hline 12 & 5.6 & 9.8 & 20.1 & 18.7 & 24.3 \\
\hline 13 & 3.8 & 7.5 & 15.9 & 25.1 & 30.7 \\
\hline
\end{tabular}

The EV charging demand depends on the vehicle usage distance. If the charging of each EV starts just after the returning home, the time-series of aggregated $\mathrm{EV}$ charging in a distribution network depends on the number of returning home EVs of different usage distance along with time. By using a Monte-Carlo simulation, the proposed model determines the number of returning home EVs in consideration of the ratio of destination zones for commuting shown in Figure 3 and the cumulative ratio of returning home from commuting shown in Figure 4.

In order to calculate the charging demand of each EV of different destination zone, the vehicle usage distance of each destination zone should be determined. In the proposed model, the distance is simply determined based on the geographical size of destination zones by assuming the square area with the same geographical size of each destination zone. Depending on the size and location of zones, the distance can be different. Table 2 shows the assumption on round-trip distance for commuting by vehicle depending on destination zone for commuting. The distance is translated to electricity consumption by assuming that the electricity consumption rate of $\mathrm{EV}$ is assumed to be $125 \mathrm{Wh} / \mathrm{km}$.

The proposed model assumes that EV is fully charged
Table 3 Setting of EV charging start time in SOC-case

\begin{tabular}{|c|c|c|c|}
\hline & \multicolumn{3}{|c|}{ SOC at returning home } \\
\hline Return time & $85 \%<$ & $75-85 \%$ & $<75 \%$ \\
\hline $6-12$ & at returning & at returning & at returning \\
\hline $12-17$ & 13 hours later & 12 hours later & 11 hours later \\
\hline $17-22$ & 7.5 hours later & 7 hours later & 6 hours later \\
\hline $22-6$ & 6 hours later & 6 hours later & at 23:00 \\
\hline
\end{tabular}

before leaving on the next day. In order to discuss the impact of charging demand profile on grid voltage, the proposed model assumes the following three cases of charging strategies:

\section{H23-case:}

Charging simultaneously starts at $11 \mathrm{pm}$ at which the night time discount electricity rate is applied.

JRH-case:

Charging starts just after the time of EV returning home.

SOC-case:

Charging starts at the time pre-determined time depending on the time of EV returning home and the SOC at that time as shown in Table 3.

The time shown in Table 3 is determined in consideration of the distribution of returning home time in Figure 4 and travel distance in Table 2.

\section{Change in Electricity Demand by EV charging}

Figure 7 shows an example of difference in EV charging demand patterns by charging strategy in winter season in distribution network located in a suburban area of Nagoya City. In H23-case, the charging demand increases significantly because charging simultaneously starts at 23:00 at which the night time discount electricity rate is applied. In JRH-case, the charging demand varies with the time of returning home of vehicles. However, the charging demand is the largest around 19:00 at which the electricity demand for other purposes is the largest. Therefore, the charging strategy in JRH-case would increase the total electricity demand in a distribution network, resulting in the decrease in voltage. In SOC-case, the charging demand varies in the mid-night, in which the electricity demand for other purposes is very small. Therefore, the charging strategy in SOC-case is expected to level the electricity demand pattern.

This study evaluates the increase in night-time electricity demand by high-penetration EV in various residential areas in Aichi Prefecture. Figure 8 shows an example of change in electricity demand by the high penetration of $\mathrm{EV}$ in winter season in distribution network located in suburban area. As a result, in H23-case, the night-time electricity demand increases much larger than the daytime peak demand without EV in all areas. The increase can be mitigated by the autonomous charging control in SOC-case.

\section{Impact on voltage in distribution network}

Finally, by using distribution network models developed according to the demand density and geographical size of 


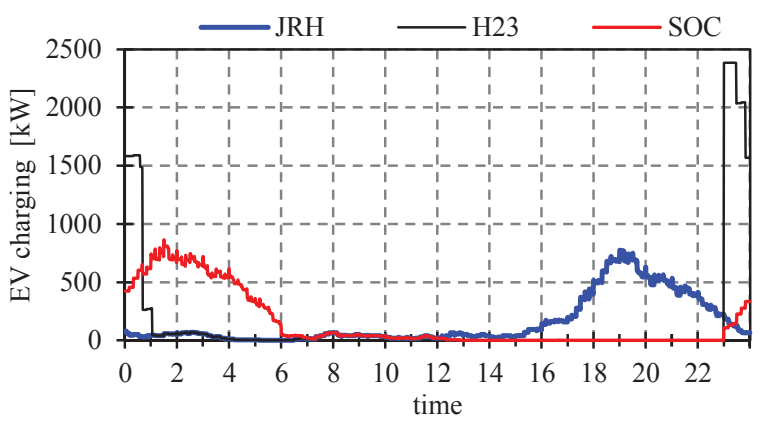

Fig. 7 Difference in EV charging demand pattern in suburban distribution network according to EV charging strategy

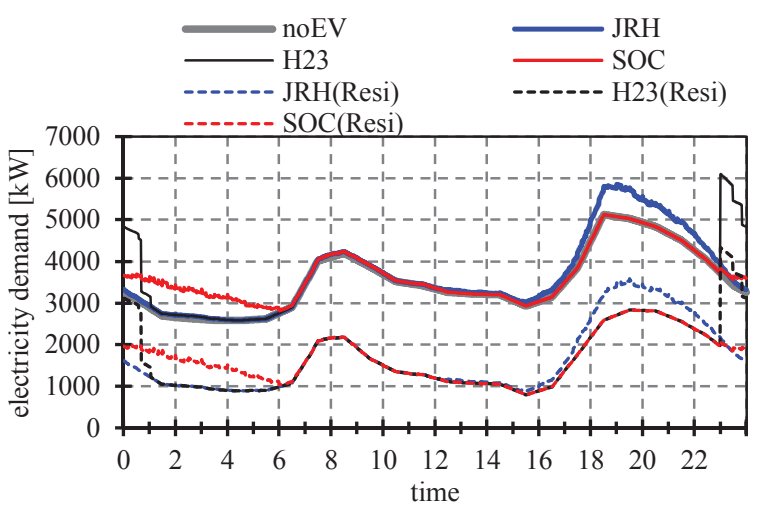

Fig. 8 Difference in electricity demand pattern including EV charging demand in suburban distribution network according to EV charging strategy

territory, this study assesses the impact of EV charging on voltage profile. Figure 9 (a) shows the topology of distribution network model used for the distribution network with the electricity demand shown in Figure 8. Circles show the demand points at which consumers in commercial sector are connected. In each point, low voltage feeder lines on low voltage side shown in Figure 9(b) is connected to supply the electricity to residences. The number of parallel lines are determined in consideration of total number of residences in the territory.

Figure 10 shows the result of voltage at the end node of distribution network model in winter season. The voltage w/o EV is the same of the highest voltage among three cases with different EV strategies. In JRH-case, the voltage decreases to $94 \mathrm{~V}$ at 19:00, because the EV charging demand is added to the original large electricity demand in the evening even though the EV charging demand varies along with the time depending on the temporal distribution of returning home. In H23-case, because EV charging demand increases significantly at 23:00, the voltage drops below $90 \mathrm{~V}$ steeply at 23:00. Compared to these cases, in SOC-case with the distributed EV charging demand shifted to the midnight, the voltage drop in the evening is mitigated. Besides, the original electricity demand in the midnight is small. Therefore, the EV charging demand in the midnight does not affect so much on the voltage profile.

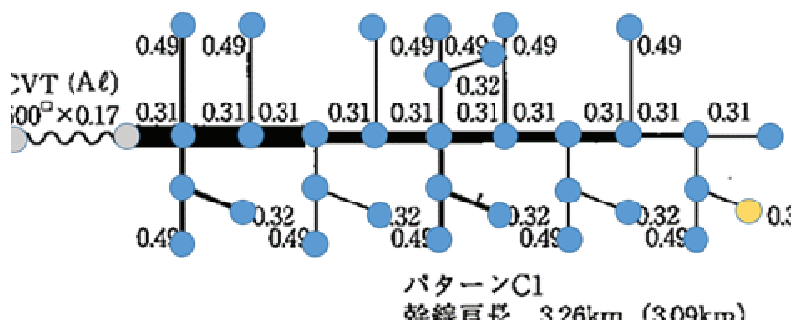

(a) topology

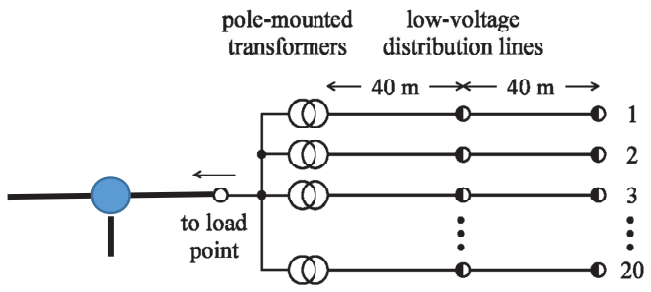

(b) assumption on feeder lines on low voltage side Fig. 9 Distribution network model

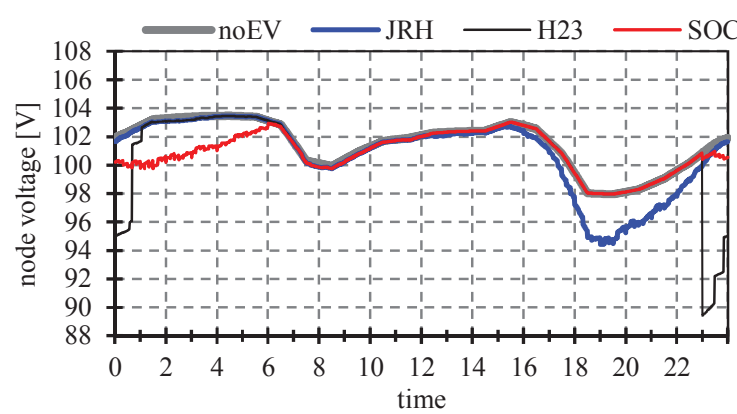

Fig. 10 Difference in voltage pattern at end-node in suburban distribution network area according to EV charging strategy

Figure 11 shows the frequency of voltage level at each time of day in winter season in distribution network in suburban area. The result shows the impact of EV charging on distribution network voltage can be avoided when the increase in electricity demand by EV charging is mitigated by the autonomous charging control.

\section{Conclusion}

For the statistical assessment of EV impact in various distribution networks, this study developed a model to estimate time-series data of electricity demand including EV charging based on Person-trip Survey Data and Grid Square Statistics Data on geographical distribution of population, employee, etc. Because the proposed model is based on the publically available statistical data, it is useful for evaluating the impact of high penetration of EV on the voltage profile in arbitrary distribution network in consideration of population change toward the future.

The proposed model was applied to a typical distribution network in suburban area of Nagoya City and discussed the EV charging strategies in three cases. The main results are as follows. If the charging simultaneously starts at 23:00 at which the night time discount electricity rate is applied, the night-time electricity demand would be much larger than the day-time peak demand without EV in most 


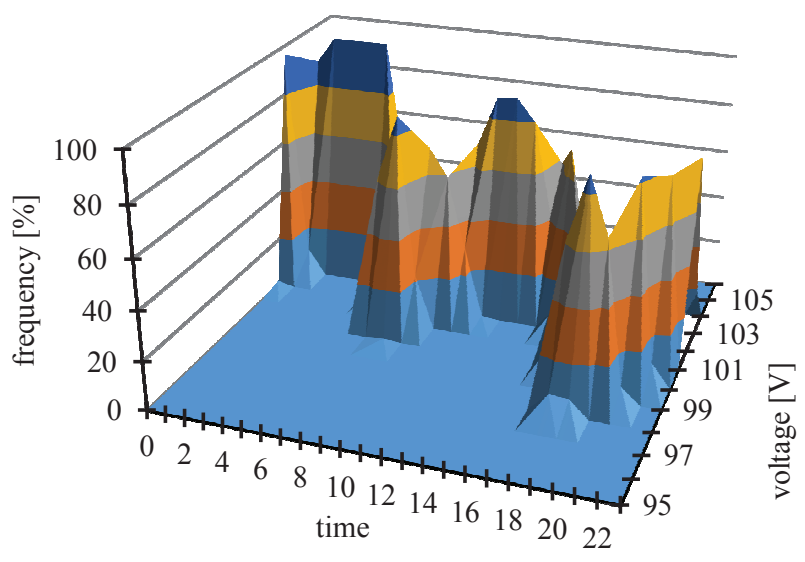

(a) w/o EV

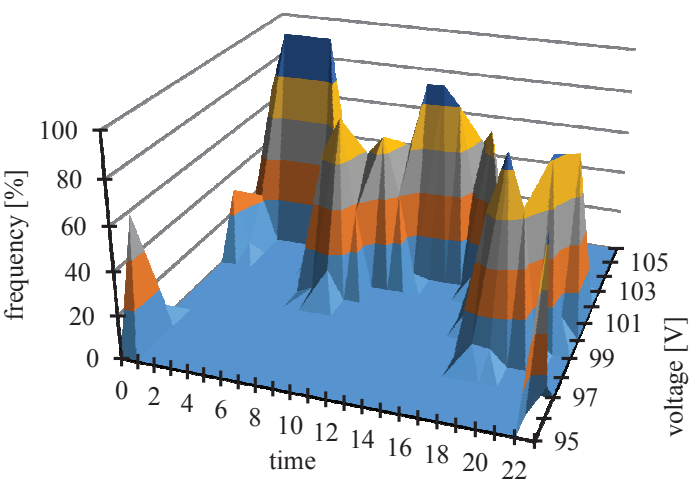

(c) H23-case

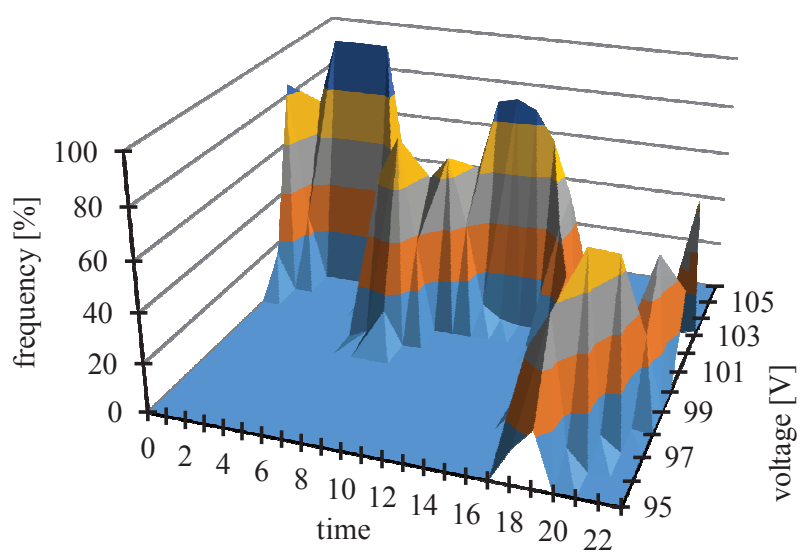

(b) JRH-case

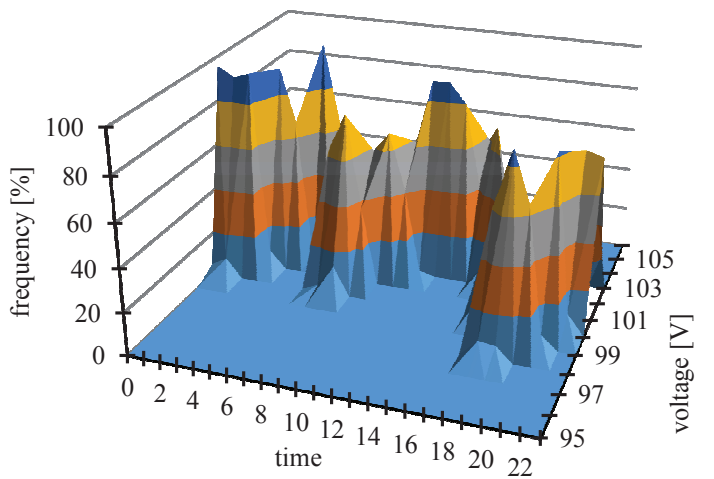

(d) SOC-case

Fig. 11 Frequency of voltage level at each time of day in winter season in distribution network in suburban area.

of distribution network. The increase would be mitigated by the autonomous charging control according to the time and SOC of EV coming back home in most areas. The impact of EV charging on voltage profile. The result shows the impact of EV charging on distribution network voltage can be avoided by applying the proposed autonomous charging control.

As a future work, the impact of various EV charging strategies will be evaluated for various distribution networks in consideration of high penetration of PV generations. For example, person-trip Survey Data shows that certain number of vehicles are not used daytime. If these vehicles are replaced with $\mathrm{EV}$, the charging of these EVs can contribute to mitigate voltage rise caused by the reverse power flow from photovoltaic power generations in distribution network. Besides, if discharging of these EVs is available, it can contribute to the power supply and demand balancing in whole electric power system. These benefits of using EV will be discussed in a future work by using the proposed demand estimation model in this study.

\section{References}

[1] T. Noda, Y. Kabasawa, K. Fukushima, Y. Nemoto, and S. Uemura, "A Method for Compensating Customer Voltage Drops due to Nighttime Simultaneous Charging of EV's Utilizing Reactive Power Injection from Battery Chargers", IEEJ Trans. PE, Vol.132, No.2, pp.163-170 (2012)
[2] Z. Akhtar, B. Chaudhuri, and S. Hui, "Smart Loads for Voltage Control in Distribution Networks", IEEE Trans. on Smart Grid, Vol.8, No.2, pp.937-946 (2017)

[3] A. T. Procopiou, J. Quiros-Tortos, and L.F. Ochoa, "HPC-Based Probabilistic Analysis of LV Networks With EVs: Impacts and Control", IEEE Trans. on Smart Grid, Vol.8, No.3, pp.1479-1487 (2017)

[4] Q. R. Hamid, and J. A. Barria, "Congestion Avoidance for Recharging Electric Vehicles Using Smoothed Particle Hydrodynamics", IEEE Trans. on Power Systems, Vol.31, pp.1014-1024 (2016)

[5] S. Faddel, O. A. Mohammed, "Automated Distributed Electric Vehicle Controller for Residential Demand Side Management", IEEE Trans on Industry Applications, Vol.55, pp.16-25 (2019)

[6] J. Kitayama, M. Kurimoto, T. Kato, Y. Suzuoki, "Statistical Evaluation of Net Electricity Load in Power Distribution Substation Supply Area with High Penetration Photovoltaic Power Generation System - Case-Study for Various Areas in Nagoya City- ", Proc. of the 30th Conf. on Energy Economy, and environment, No.25-3, pp.475-480 (2014) (in Japanese)

[7] J. Kitayama, M. Kurimoto, T. Kato, Y. Suzuoki: "Aggregated Photovoltaic Power Output Fluctuation Characteristics in Power System Considering Power supply Limitation in Each Power Distribution Area",Proc. of the 31st Conf. on Energy, Economy, and Environment, No.3-3, pp.51-56 (2015) (In Japanese) 